\title{
Spike-Time Precision and Network Synchrony Are Controlled by the Homeostatic Regulation of the D-Type Potassium Current
}

\author{
Robert H. Cudmore, Laure Fronzaroli-Molinieres, Pierre Giraud, and Dominique Debanne \\ Inserm U641, Faculté de Médecine Secteur Nord, Université de la Méditerranée, 13344 Marseille, France
}

\begin{abstract}
Homeostatic plasticity of neuronal intrinsic excitability (HPIE) operates to maintain networks within physiological bounds in response to chronic changes in activity. Classically, this form of plasticity adjusts the output firing level of the neuron through the regulation of voltage-gated ion channels. Ion channels also determine spike timing in individual neurons by shaping subthreshold synaptic and intrinsic potentials. Thus, an intriguing hypothesis is that HPIE can also regulate network synchronization. We show here that the dendrotoxin-sensitive D-type $\mathrm{K}^{+}$current $\left(I_{\mathrm{D}}\right)$ disrupts the precision of AP generation in CA3 pyramidal neurons and may, in turn, limit network synchronization. The reduced precision is mediated by the sequence of outward $I_{\mathrm{D}}$ followed by inward $\mathrm{Na}^{+}$current. The homeostatic downregulation of $I_{\mathrm{D}}$ increases both spike-time precision and the propensity for synchronization in iteratively constructed networks in vitro. Thus, network synchronization is adjusted in area CA3 through activity-dependent remodeling of $I_{\mathrm{D}}$.
\end{abstract}

\section{Introduction}

It is now well established that chronic activity regimens in neuronal circuits induce a homeostatic plasticity of intrinsic excitability (HPIE) that regulates voltage-gated currents (Turrigiano et al., 1994; Desai et al., 1999). It is generally thought that this form of plasticity acts to adjust the output firing level of the postsynaptic neuron to stabilize network activity within physiological bounds. The function of voltage-gated ion channels is, however, not purely limited to the control of neuronal excitability. In parallel to properties of synaptic connections (Pouille and Scanziani, 2001; Buonomano, 2005; Boudkkazi et al., 2007), ion channels also contribute to spike-time precision and network synchronization (Schneidman et al., 1998; Schoppa and Westbrook, 1999). An important and entirely novel issue is whether homeostatic plasticity of neuronal excitability can also determine spike timing and sculpt network synchrony.

The fast-on, slow-off D-type voltage-gated $\mathrm{K}^{+}$current $\left(I_{\mathrm{D}}\right)$ has been shown to create a delay in the onset of the first action potential (AP) and to, in part, define intrinsic excitability in a number of neuronal types, including CA1 pyramidal neurons of the hippocampus (Storm, 1988), L5 (Bekkers and Delaney, 2001;

Received Feb. 9, 2010; revised July 8, 2010; accepted July 28, 2010.

This work was supported by Inserm Avenir (D.D.), Centre National de la Recherche Scientifique, Agence Nationale de la Recherche (Neurosciences, neurologie et psychiatrie 06-Neuro-014-01 to D.D.), Inserm/International Brain Research Organization and Association Française contre les Myopathies postdoctoral grants (R.H.C.), and European Community Grant LSHM-CT-2004-511995 (D.D.). We thank G. Alcaraz, N. Ankri, E. Campanac, O. Caillard, M. Russier, and L. Savtchenko for helpful discussions and N. Ankri for help with some experiments. We also thank R. Miles, I. Segev, B. Rudy, D. Linden, M. Seagar, and F. Tell for constructive feedback on this manuscript.

Correspondence should be addressed to Dominique Debanne at the above address. E-mail: dominique. debanne@univmed.fr.

R. H. Cudmore's present address: The Solomon H. Snyder Department of Neuroscience, The Johns Hopkins University, Baltimore, MD 21205.

DOI:10.1523/JNEUROSCI.0740-10.2010

Copyright $\odot 2010$ the authors $\quad 0270-6474 / 10 / 3012885-11 \$ 15.00 / 0$
Kole et al., 2007; Shu et al., 2007; Miller et al., 2008), and L2-3 (Guan et al., 2007) pyramidal neurons of the cortex and L2-3 fast-spiking interneurons of the somatosensory cortex (Goldberg et al., 2008). Given that $I_{\mathrm{D}}$ is activated at voltages near AP threshold (Wu and Barish, 1992; Locke and Nerbonne, 1997) and is located primarily on the soma and axon (Kole et al., 2007; Shu et al., 2007), it has a potentially critical role in determining the transformation from synaptic input into AP output.

One mechanism by which outward $\mathrm{K}^{+}$current can increase AP precision is by a sequence of (1) inward $\mathrm{Na}^{+}$current to generate an AP followed by (2) outward $\mathrm{K}^{+}$current to limit additional APs (Fricker and Miles, 2000; Axmacher and Miles, 2004; Campanac et al., 2008). This is conceptually similar to the improvement of AP precision by the sequence of inward synaptic excitation followed by outward synaptic inhibition (Pouille and Scanziani, 2001). But outward $\mathrm{K}^{+}$current can also decrease AP precision. For instance, activation of SK channels by the first AP reduces the rate of depolarization leading to the second spike and increases its temporal jitter (Sourdet et al., 2003). Thus, a clarification of the rules by which outward $\mathrm{K}^{+}$currents sculpt AP precision is needed.

We show here that the expression of a dendrotoxin (DTX)sensitive outward $\mathrm{K}^{+}$current $\left(I_{\mathrm{D}}\right)$ desynchronizes APs in individual neurons and, in turn, reduces network synchronization. Furthermore, we show that the reduction in $I_{\mathrm{D}}$ after HPIE can increase both spike precision and network synchronization. We conclude that in addition to maintaining functional firing rates, HPIE is able to sculpt network synchrony by modulating postsynaptic ion channels.

\section{Materials and Methods}

Organotypic hippocampal slice cultures. Hippocampal slice cultures were prepared using an interface technique (Stoppini et al., 1991; Debanne et al., 2008). Briefly, postnatal day 5-7 Wistar rats were deeply anesthetized by intraperitoneal injection of chloral hydrate, the brain was removed, 
and each hippocampus was individually dissected. Hippocampal slices $(300 \mu \mathrm{m})$ were placed on $20 \mathrm{~mm}$ latex membranes (Millicell) inserted into $35 \mathrm{~mm}$ Petri dishes containing 1 $\mathrm{ml}$ of culture medium and maintained for up to $21 \mathrm{~d}$ in an incubator at $34^{\circ} \mathrm{C}, 95 \% \mathrm{O}_{2}-5 \%$ $\mathrm{CO}_{2}$. The culture medium contained (in $\mathrm{ml}$ ) 25 MEM, 12.5 HBSS, 12.5 horse serum, 0.5 penicillin/streptomycin, 0.8 glucose $(1 \mathrm{M}), 0.1$ ascorbic acid $(1 \mathrm{mg} / \mathrm{ml}), 0.4$ HEPES (1 M), 0.5 B27, and 8.95 sterile $\mathrm{H}_{2} \mathrm{O}$. To arrest glial proliferation, $5 \mu \mathrm{m}$ Ara- $\mathrm{C}$ was added to the culture medium starting at 3 days in vitro (DIV).

Electrophysiology. Slice cultures were placed in a perfusion chamber on an upright microscope for visual identification using infrared differential interference contrast optics. Wholecell recordings from CA3 pyramidal neurons were performed at $34^{\circ} \mathrm{C}$ using a Multiclamp 700B or Axopatch 200B amplifier (Molecular Devices). Recordings were analog filtered at $5-7 \mathrm{kHz}$ and digitized at $20 \mathrm{kHz}$ (NIDAQ and DigiData 1322A; National Instruments and Molecular Devices). Experiments were discarded if the resting membrane potential $\left(V_{\mathrm{m}}\right)$ changed by $>6 \mathrm{mV}$ or the resting input resistance (measured as the response to a -20 pA hyperpolarizing DC pulse) changed by $>10 \%$. In voltage clamp, series resistance (range, 15-20 M $\Omega$ ) and whole-cell capacitance were measured by fitting the response to a DC voltage command $(5 \mathrm{mV}, 300 \mathrm{~ms})$; recordings were discarded if the series resistance became $>40$ $\mathrm{M} \Omega$. All analyses were performed with custom-written programs using Igor Pro 6 (Wavemetrics).

Solutions and pharmacology. The control perfusion solution contained (in $\mathrm{mM}$ ) 125 $\mathrm{NaCl}, 26 \mathrm{NaHCO}_{3}, 3 \mathrm{CaCl}_{2}, 2.5 \mathrm{KCl}, 2 \mathrm{MgCl}_{2}$, $0.8 \mathrm{NaH}_{2} \mathrm{PO}_{4}$, and 10 D-glucose and was continuously equilibrated with $95 \% \mathrm{O}_{2}-5 \% \mathrm{CO}_{2}$. Patch pipettes (6-9 $\mathrm{M} \Omega$ ) were filled with a solution containing (in $\mathrm{mM}$ ) $120 \mathrm{~K}$-gluconate, 20 $\mathrm{KCl}, 10$ HEPES, 0.5 EGTA, $2 \mathrm{Na}_{2} \mathrm{ATP}, 0.3$ $\mathrm{NaGTP}$, and $2 \mathrm{MgCl}_{2}, \mathrm{pH}$ 7.4. All drugs were applied via the bath solution. In most experiments, fast synaptic transmission was blocked using 2-4 mm kynurenate (Ky) and $100 \mu \mathrm{m}$ picrotoxin (PiTX). To block voltage-dependent $\mathrm{Ca}^{2+}$ and fast $\mathrm{Na}^{+}$channels and isolate $\mathrm{Kv}$ currents, the following was added to the control perfusion solution: $200 \mu \mathrm{M} \mathrm{Ni}^{2+}, 125 \mu \mathrm{M}$ $\mathrm{Cd}^{2+}$, and $0.5 \mu \mathrm{M}$ tetrodotoxin (TTX). D-type currents (Kv1 channels) were blocked by bath application of low concentrations of 4-aminopyridine (4-AP; 20-30 $\mu \mathrm{M})$, DTX-I

(50-100 nM; Latoxan), or DTX-K (50-100 nM; Sigma) to block Kv1.1/2/6 and Kv1.1 channels, respectively. To chronically block excitatory transmission, 2-4 mм Ky was added to the culture medium at 5 DIV and replenished every $2 \mathrm{~d}$; recordings of treated cultures were performed 7-9 DIV.

Experimental protocol and data analysis. Intrinsic excitability was measured in current clamp by delivering a range of DC step pulses (50-600 pA, $1.0 \mathrm{~s}$ ) at an interstimulus interval of $10-15 \mathrm{~s}$ (Cudmore and Turrigiano, 2004). The AP threshold was measured as the interpolated time when the derivative of $V_{\mathrm{m}}$ equals $20 \mathrm{mV} / \mathrm{ms}$; this was then used as the time of the AP. The slope of the membrane potential trajectory before the AP $(\mathrm{dV} / \mathrm{dt})$ was measured in a time window $5-10 \mathrm{~ms}$ before the AP. All statistics are reported as the mean $\pm \mathrm{SE}$, and unless otherwise noted, all statistical tests are done with the Student's $t$ test with a significance level of 0.05 .
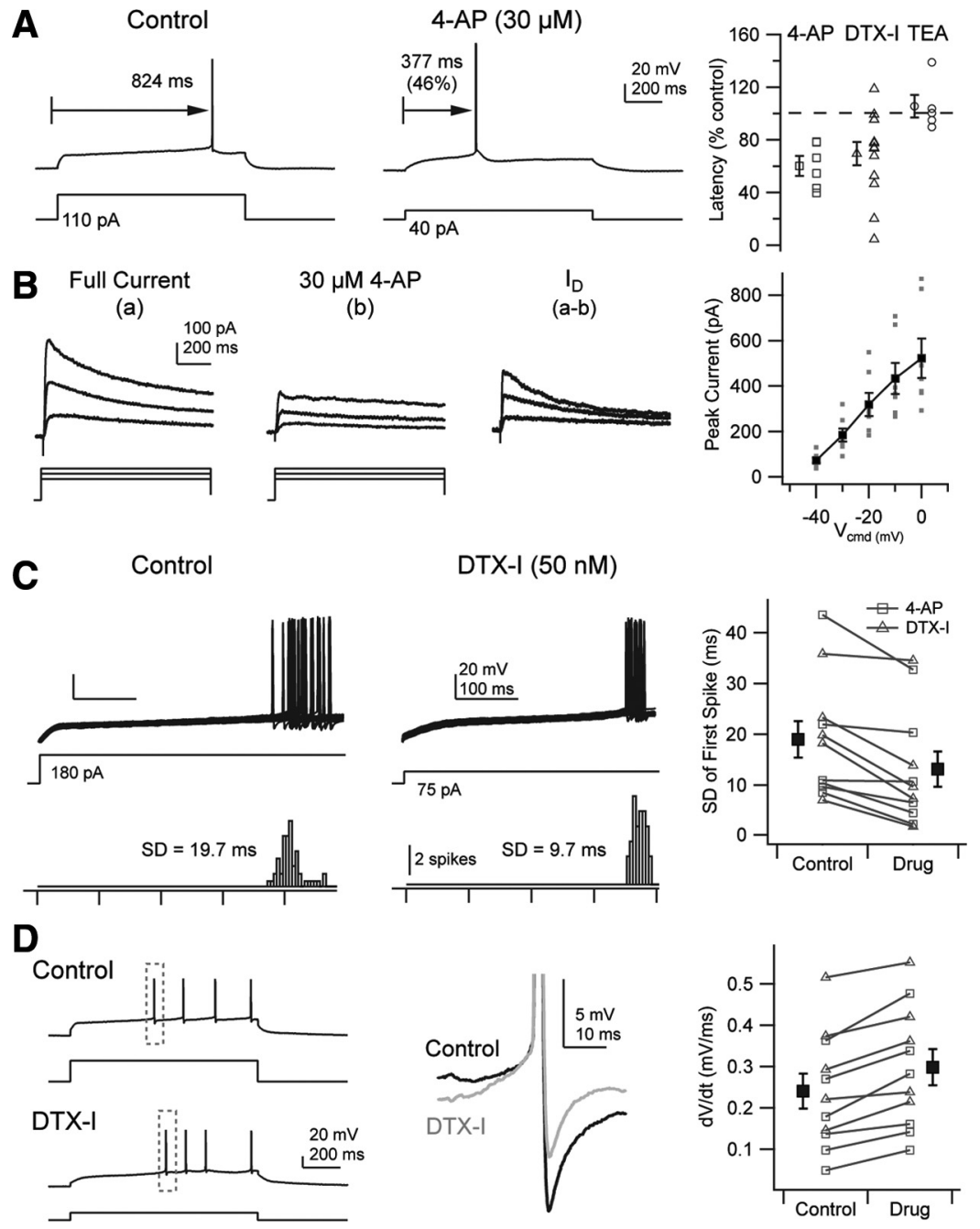

Figure 1. CA3 pyramidal neurons in organotypic slice culture express an outward $\mathrm{K}^{+}$conductance that creates a ramp-andns. Left, Example of reduced ramp-and-delay phenotype induced by low concentration of 4-AP (30 $\mu \mathrm{m})$. Note the decrease in the to the first spike in the presence of 4-AP (46\% of the control). Right, Summary of the normalized delay to the first spike

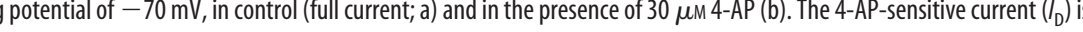
(

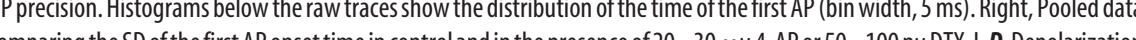
作 depolarization rate (dV/dt) induced by the blockade of $I_{D}$ with 4-AP (squares) or DTX-I (triangles).

Voltage-clamp protocols to measure Kv currents consisted of a family of voltage-step commands from a holding potential of $-70 \mathrm{mV}(-40$ to $+20 \mathrm{mV}$ in $10 \mathrm{mV}$ increments; $1 \mathrm{~s})$. Algebraic isolation of $I_{\mathrm{D}}$ was done by subtracting the current evoked in the presence of $I_{\mathrm{D}}$ channel blockers (4-AP, DTX-I) from currents evoked in control solution. The peak current amplitude for each voltage command was calculated from the average of 5-15 traces. In all voltage-clamp experiments, leak subtraction was performed using a $\mathrm{p} / \mathrm{n}(n=4)$ protocol, and series resistance was not compensated.

Simulated synaptic waveforms were generated with a double exponential function, $f(t)=a \cdot\left(1-\exp \left(-t / \tau_{o n}\right)\right) \cdot \exp \left(-t / \tau_{\text {off }}\right)$, where $a$ is the amplitude and $\tau_{o n}$ and $\tau_{\text {off }}$ specify the onset and decay time constants. For voltage-clamp recordings, this was injected to simulate an EPSP with an 
A Control
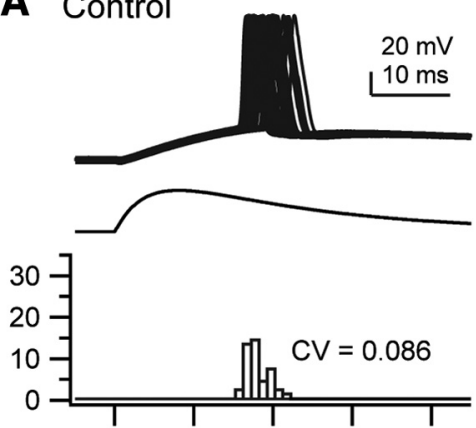

B

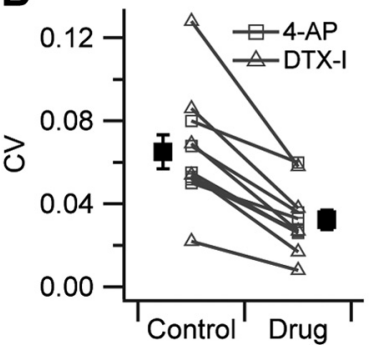

C
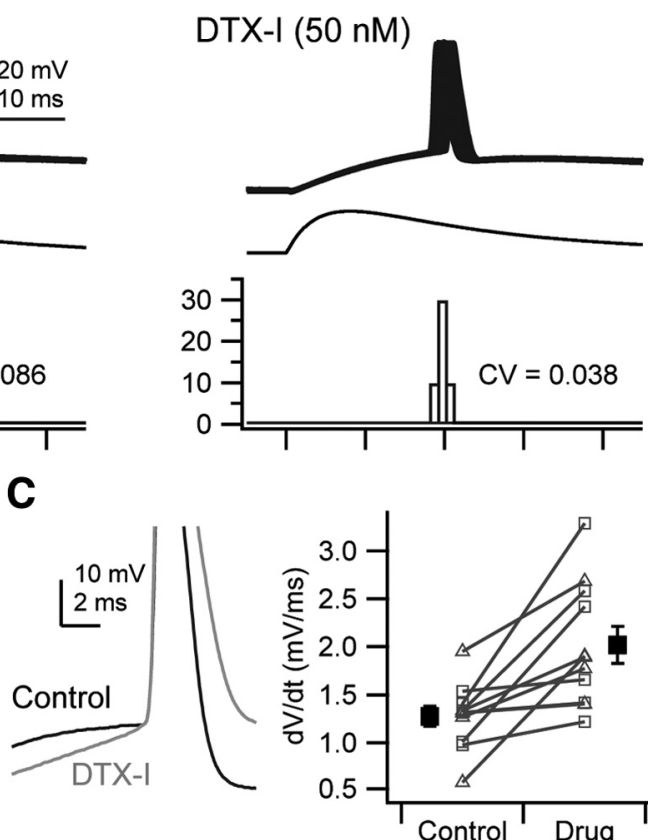

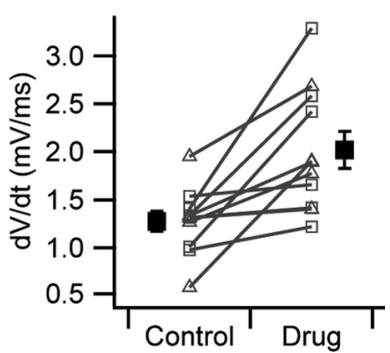

Figure 2. I controls spike time precision in response to synaptic-like input. $\boldsymbol{A}$, Example current-clamp traces of APs evoked by stimulation with dEPSCS, showing significantly more jitter in AP generation in control (left) versus DTX-I (right). Histograms below the raw traces show the distribution of the spike time. Right, Pooled data comparing the CV of the first AP onset time in control and in the presence of $30 \mu \mathrm{m}$ 4-AP or DTX-I. B, Blocking $I_{D}$ increases the derivative of the membrane potential (dV/dt) before the AP. Left, Representative traces. Right, Group data. C, Improvement of precision quantified by $\delta \mathrm{dV} / \mathrm{dt}$ is inversely correlated with the variation in voltage trajectory.

amplitude of $10-20 \mathrm{mV}$ with $\tau_{\text {on }}=10 \mathrm{~ms}$ and $\tau_{\text {off }}=50 \mathrm{~ms}$. For currentclamp recording, the double exponential function of conductance, $f(t)$, was converted to a current, $I(t)=f(t) \cdot\left(V_{\mathrm{m}}(t)-V_{\mathrm{k}}\right)$, using real-time dynamic clamp (SM1; Cambridge Conductance), and injected to simulate an excitatory postsynaptic current [dynamic-clamp EPSC (dEPSC)] where $V_{\mathrm{m}}$ is the recorded membrane potential and $V_{\mathrm{k}}$ is the reversal potential $(0 \mathrm{mV})$. In current clamp, a range of amplitudes was injected to evoke both subthreshold and suprathreshold responses ( $a$ in the range of $50-400 \mathrm{pA}, \tau_{\text {on }}=1 \mathrm{~ms}, \tau_{\text {off }}=10 \mathrm{~ms}$ ).

Stochastic Hodgkin-Huxley model. A compartmental Hodgkin-Huxley model was implemented in NEURON (version 6.2, available at http:// www.neuron.yale.edu/neuron/) using the previously published CA3 model of Hemond et al. (2008). Three different model morphologies were verified to exhibit the same qualitative behavior: (1) a fully reconstructed CA3 neuron, (2) a reduced reconstructed CA3 neuron, and (3) a ball-and-stick CA3 neuron. The simulations presented here are from the ball-and-stick implementation. The geometry consisted of a soma, basal and apical dendrites, and an axon (supplemental Table S1, available at www.jneurosci.org as supplemental material). The model included the following conductance-based currents with conductance $(g)$ densities given in siemens per square centimeter: $\mathrm{a} \mathrm{Na}^{+}$current for AP generation $\left(g_{\mathrm{Na}}=0.022\right)$, a delayed rectifier $\mathrm{K}^{+}$current $\left(g_{\mathrm{KDr}}=0.014\right)$, a Ca ${ }^{2+}$ current $\left(g_{\text {ca }}=0.001\right)$, a fast transient A-type $\mathrm{K}^{+}$current $\left(g_{\mathrm{A}}=0.02\right)$, a fast-activating, slow-offset D-type $\mathrm{K}^{+}$current $\left(g_{\mathrm{D}}=0.0011\right)$, and a leak current $\left(g_{\text {pas }}=3.9 \mathrm{e}-5\right)$. Current densities were uniformly distributed in all compartments, and the model was tested to give similar results within a range of these current densities. Each current was specified as a firstorder differential equation following Hodgkin and Huxley formalisms and were identical to the equations in Hemond et al. (2008). The $\mathrm{Na}^{+}$ channel density was increased 10 -fold in the axon, and it was verified that APs were initiated in the axon. The model was run at $35^{\circ} \mathrm{C}$, the membrane capacitance was $1.41 \mathrm{uF} / \mathrm{cm}^{2}$, and the time step was $0.0125 \mathrm{~ms}$.

To introduce channel noise, a stochastic $\mathrm{Na}^{+}$channel was implemented using the NEURON-extension NMODL (Hines and Carnevale, 2000) based on the deterministic $\mathrm{Na}^{+}$channel (Hemond et al., 2008). The stochastic $\mathrm{Na}^{+}$channel was implemented as an approximation to a full Markov model following the algorithm of Fox (1997). For each compartment, the number of $\mathrm{Na}^{+}$channels was calculated as $N_{\mathrm{Na}}=$ area ${ }^{*} g_{\mathrm{Na}} / \gamma$, where area is in square centimeters, $g_{\mathrm{Na}}$ is in siemens per square centimeter, and the unitary channel conductance is $\gamma=20$ pS. For each gating particle $x=\{\mathrm{m}, \mathrm{h}, \mathrm{s}\}$, a random voltage-dependent noise was added at each time step by selecting from a Gaussian probability density function with mean $\mu(t)=$ 0 and variance $\sigma(t)^{2}$ given by the following:

$$
\sigma_{\mathrm{x}}^{2}(t)=\frac{2}{N_{\mathrm{Na}}} \frac{\alpha_{\mathrm{x}}(t) \beta_{\mathrm{x}}(t)}{\alpha_{\mathrm{x}}(t)+\beta_{\mathrm{x}}(t)}
$$

Hybrid network model. Layered feed-forward hybrid networks were constructed using a real neuron and simulated synapses (Reyes, 2003). To construct a network of $n$ layers with $m$ neurons per layer, the first layer was modeled as $m$ artificial neurons firing APs at an average frequency $(10 \mathrm{~Hz}, 1 \mathrm{~s})$. To introduce jitter in this modeled layer, each AP and each AP train was randomly shifted in time by $10 \%$ of the mean interspike interval. The binary AP output of this simulated layer was convolved with a template EPSC (same parameters as current-clamp experiments); this then became the presynaptic input to the first layer. A single whole-cell recording was then used to construct the remaining hybrid network. Each neuron in the hybrid network received a current injection (at intervals of $10 \mathrm{~s}$ ) constructed from the summation of a random percentage $(40 \%)$ of the convolved presynaptic activity. To simulate synaptic noise, the template EPSC amplitudes and timing were chosen from a Gaussian distribution with a mean and SD of $40 \pm 10 \mathrm{pA}$ and $0.1 \pm 1 \mathrm{e}-5 \mathrm{~ms}$, respectively (Debanne et al., 1995). To construct a five-layer hybrid network took 2-3 h. The quality of the recording was confirmed by monitoring the stability of the resting membrane potential and input resistance. To make meaningful comparisons of synchrony across networks in different conditions, great care was taken to maintain stable resting membrane potentials and similar firing rate outputs. The synchrony within each layer was quantified as the 0 time-lag peak in the normalized and summed pairwise cross-correlation histograms (CCHs) of the binary spike trains of each neuron (bin width, 2 ms) (Reyes, 2003; Wang and Buzsaki, 1996).

\section{Results}

\section{$I_{\mathrm{D}}$ current reduces AP precision in CA3 pyramidal neurons}

CA3 pyramidal neurons analyzed by whole-cell recording in organotypic slice cultures exhibited a voltage-dependent subthreshold ramp and a long delay to the firing of the first AP (Fig. $1 A)$. The ramp-and-delay phenotype could be abolished by voltage inactivation or bath application of the $\mathrm{K}^{+}$channel blocker 4-AP or DTX-I. On average, the reduction in the delay to the first spike onset was significantly reduced in the presence of either 4 -AP $(30 \mu \mathrm{M})$ or DTX-I ( $50 \mathrm{nM}$ ) (control, $765 \pm 35 \mathrm{~ms}, n=25 \mathrm{vs}$ 4 -AP, $436 \pm 54 \mathrm{~ms}, n=6, p=0.03$, and DTX-I, $514 \pm 73 \mathrm{~ms}, n=$ $13, p=0.0007$ ) (Fig. $1 A$ and supplemental Table S2, available at www.jneurosci.org as supplemental material). No change in resting membrane potential was induced by the drugs $(-69.7 \pm 1.6$ $\mathrm{mV}$ in control vs $-69.7 \pm 1.5 \mathrm{mV} ; p=0.8)$. In contrast, the channel blocker tetraethylammonium (TEA; $5 \mathrm{~mm}$ ) had no effect (Fig. $1 \mathrm{~A}$ and supplemental Fig. S1, available at www.jneurosci. org as supplemental material $)(807 \pm 136 \mathrm{~ms}$ vs $831 \pm 15 \mathrm{~ms} ; n=$ 5 ; paired $t$ test, $p=0.76$ ). Together, these results suggest the delay to the first spike is mediated by a transient outward $\mathrm{K}^{+}$current 
similar to an $I_{\mathrm{D}}$-like current (Storm, 1988; Luthi et al., 1996; Mitterdorfer and Bean, 2002; Hemond et al., 2008) present in CA3 pyramidal neurons in acute slices (supplemental Fig. S2, available at www.jneurosci. org as supplemental material).

To examine the outward currents activated in response to depolarizing input, voltage-clamp recordings were performed in the presence of synaptic and ion channel blockers (see Materials and Methods). In control recordings, a transient outward current with slow inactivation was evoked in response to a range of DC steps (Fig. $1 B$ ) (inactivation time constant at -20 $\mathrm{mV}$ was $2.7 \mathrm{~s}$ ). Bath application of low concentrations of 4-AP blocked the transient outward current, and algebraic subtraction of the current evoked in control versus in the presence of 4-AP revealed an $I_{\mathrm{D}}$-like current, which first activates at -40 $\mathrm{mV}$, before the activation of inward $\mathrm{Na}^{+}$ currents. In addition, a fast transient A-type current could be isolated with higher concentration of 4-AP (supplemental Fig. S3, available at www.jneurosci.org as supplemental material).

To determine how the ramp-anddelay phenotype mediated by the inactivation of $I_{\mathrm{D}}$ affects $\mathrm{AP}$ precision, we measured the SD of the latency of the first $\mathrm{AP}$ in response to repeated DC current injections. In control conditions, onset of the first AP occurred with relatively low precision (Fig. $1 C$ ). In contrast, pharmacological reduction of $I_{\mathrm{D}}$ by bath application of 4-AP $(30 \mu \mathrm{M})$ or DTX-I (50-100 $\mathrm{nM})$ significantly increased the precision of the first AP (SD in control: $19.0 \pm 3.7$ $\mathrm{ms}$ vs in the presence of D-type channel blockers: $13.2 \pm 3.6 \mathrm{~ms} ; n=11 ; p=$ 0.0007) (supplemental Table S2, available at www.jneurosci.org as supplemental material). To make meaningful comparisons of AP precision between conditions, the delay to the first AP and evoked firing frequency were kept constant by adjusting the amplitude of the injected current. Concomitant with the increase in precision, the reduction of $I_{\mathrm{D}}$ also increased the voltage slope just before (5-10 ms window) the first AP (control, $0.24 \pm 0.04 \mathrm{mV} / \mathrm{ms}$; D-type channel blockers $0.30 \pm 0.04 \mathrm{mV} / \mathrm{ms} ; n=11 ; p=0.00008$ ) (Fig. $1 D$ ). An alternative to pharmacological inactivation of $\mathrm{Kv}$ currents is voltage inactivation. Reducing Kv currents by holding the membrane potential at $-55 \mathrm{mV}$ also improved AP precision (SD of first AP onset time: control, $35.0 \pm 4.7 \mathrm{~ms}$; inactivation, $17.7 \pm 7.0 \mathrm{~ms}$; $n=6 ; p=0.028$; data not shown). Thus, $I_{\mathrm{D}}$ introduces jitter in the timing of APs by reducing the membrane potential trajectory immediately preceding the spike.

To determine whether the presence of $I_{\mathrm{D}}$ can reduce AP precision in response to synaptic-like input, dEPSC waveforms were repeatedly injected to measure the coefficient of variation $(\mathrm{CV})$ of the first AP onset time in the presence of and after blocking $I_{\mathrm{D}}$
(Fig. 2A). Injection of suprathreshold dEPSC waveforms produced APs with more precision after the reduction of $I_{\mathrm{D}}$ with DTX-I or 4-AP (Fig. $2 \mathrm{~B}$ and supplemental Table S2, available at www.jneurosci.org as supplemental material) (CV control, $0.065 \pm 0.008$; CV after blockade of $I_{\mathrm{D}}, 0.032 \pm 0.005 ; n=11 ; p=$ $0.00005)$. The voltage trajectory just before the AP was also reduced in the presence of $I_{\mathrm{D}}$ and increased when $I_{\mathrm{D}}$ was pharmacologically blocked (Fig. 2C) (dV/dt: control, $1.27 \pm 0.1 \mathrm{mV} / \mathrm{ms}$; after blockade of $\left.I_{\mathrm{D}}, 2.02 \pm 0.2 \mathrm{mV} / \mathrm{ms} ; n=11 ; p=0.0026\right)$. Blocking both A- and D-type currents with voltage inactivation improved the precision and increased $\mathrm{dV} / \mathrm{dt}$ of the first spike in a way similar to blocking D-type current alone (supplemental Fig. S4, available at www.jneurosci.org as supplemental material) (CV in control, $0.65 \pm 0.1 \mathrm{~ms}$ vs voltage inactivated, $0.37 \pm 0.1 \mathrm{~ms} ; n=7$; $p=0.003)$. Furthermore, bath application of a high concentration of 
A Control

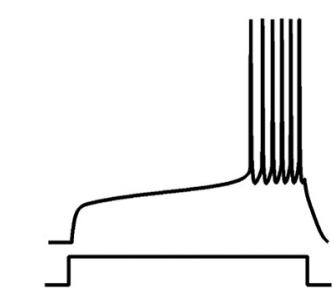

B
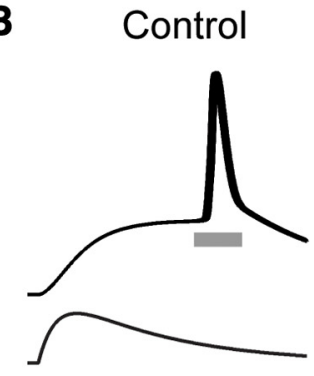

c
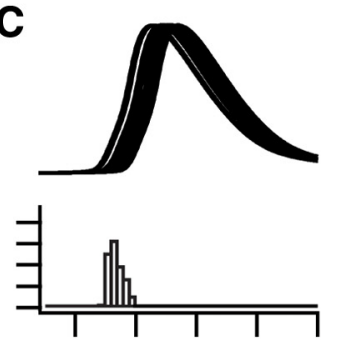

D Control

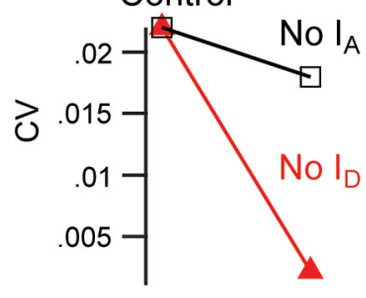

No $I_{D}$

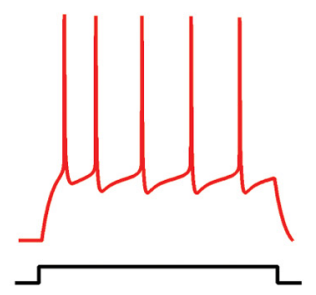

No $I_{D}$
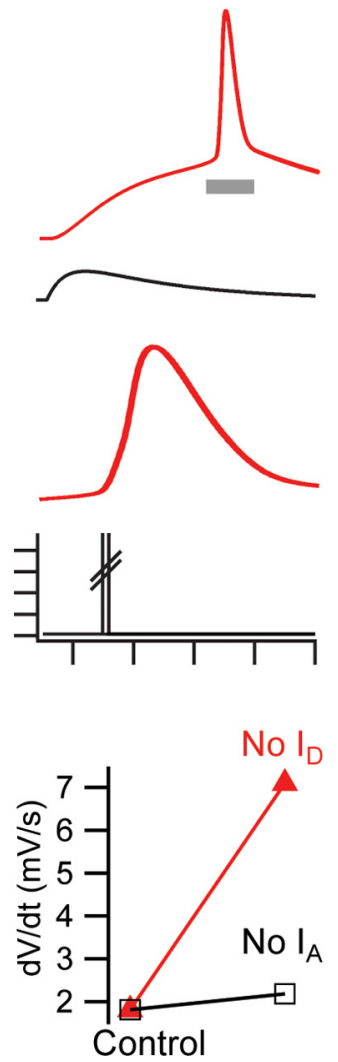
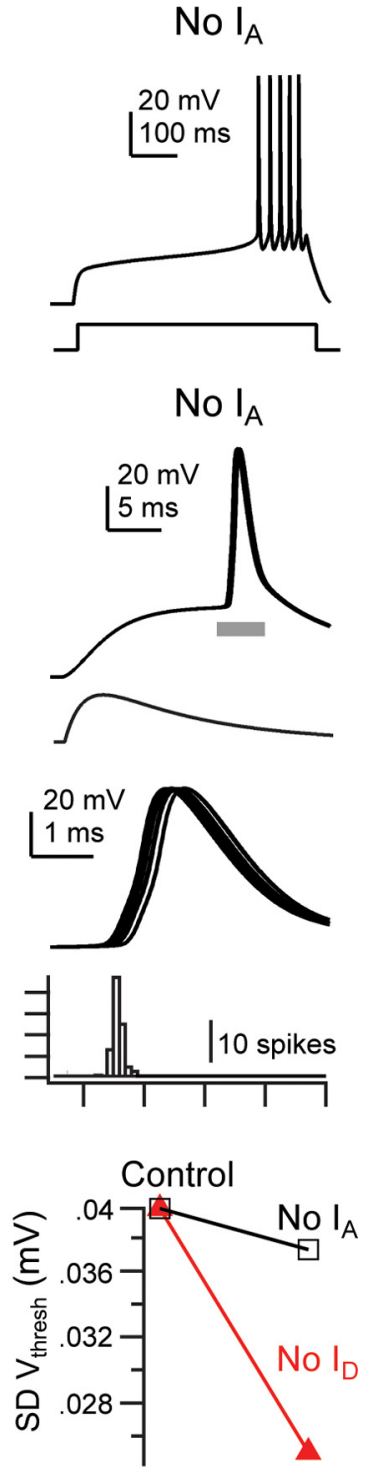

Figure 4. Stochastic Hodgkin-Huxley model of a CA3 pyramidal neuron reproduces the experimental observations. $A$, Example model response to a $D C$ current step showing the $I_{D}$-dependent delay firing phenotype (left, control). The delay in firing is reduced when $I_{D}$ (middle, № $I_{D}$ ) but not $I_{A}$ (right, № $I_{A}$ ) is removed from the model. $B$, Example model response $(n=5$ trials) to a synaptic-like EPSC waveform with (left, control) and without (middle, № $I_{D}$ ) $I_{D}$ and without $I_{A}$ (right, № $I_{A}$ ). $C$, Expanded view (horizontal gray bars in $E$ ) showing the reduction in the jitter of AP generation in response to an EPSC when $I_{D}$ but not $I_{A}$ is removed. The histogram below shows the binned AP time for the three conditions ( $n=100$ trials). $\boldsymbol{D}$, Average CV (same runs as in $\boldsymbol{B}$ and $\boldsymbol{C}$ ) is reduced when $I_{D}$ but not $I_{A}$ is removed (left; $n=100$ trials). The membrane potential trajectory (dV/dt) just before AP generation increases after removal of $I_{D}$ and is relatively unchanged on removal of $I_{A}$ (center). Finally, removal of $I_{D}$ but not $I_{A}$ reduces the variation in the voltage threshold of the AP (right; SD $V_{\text {thresh }}$ ).

4-AP (3-5 mM) improved the precision of the first AP (CV control, $0.76 \pm 0.1 \mathrm{~ms} ; 4$-AP, $0.38 \pm 0.02 \mathrm{~ms} ; n=7 ; p=0.0014)$. Thus, blocking both $I_{\mathrm{A}}$ and $I_{\mathrm{D}}$ have similar effects to blocking $I_{\mathrm{D}}$ alone, indicating a predominant role for $I_{\mathrm{D}}$ in controlling AP precision.

The sequence of outward and inward currents evoked by synaptic input greatly determines the pattern and precision of AP generation (Fricker and Miles, 2000; Vervaeke et al., 2006). We therefore characterized the sequence of currents activated by depolarizing steps and synaptic-like EPSP voltage commands in CA3 neurons. Neurons displaying delayed spiking in currentclamp recordings also exhibited a transient outward current that preceded inward current in voltage clamp (Fig. 3A). Bath application of 4-AP reduced the outward current, unmasked an in-

ward current, and abolished the delayed spiking in both current and voltage clamp. In contrast with previous findings in CA1 neurons (Fricker and Miles, 2000), subthreshold EPSPs in CA3 neurons evoked outward currents (supplemental Fig. S5, available at www.jneurosci.org as supplemental material) (CA3 neurons, $+76 \pm$ $13.8 \mathrm{pA}, n=7$ vs CA1 neurons, $-27 \pm 3.8$ $\mathrm{pA}, n=7)$. The outward current in CA3 neurons could be reduced with either voltage inactivation $(65.5 \pm 7.7 \mathrm{pA}$ in control and $14.9 \pm 10.1 \mathrm{pA}$ during voltage inactivation; $n=8$ ) or a low concentration of $4-\mathrm{AP}(82.9 \pm 18.4 \mathrm{pA}$ in control and $6.0 \pm 2.9 \mathrm{pA}$ in the presence of $20 \mu \mathrm{M}$ 4 -AP; $n=6)$ or DTX-I (65.4 $\pm 11.2 \mathrm{pA}$ in control and $18.1 \pm 6.3 \mathrm{pA}$ in the presence of 50 nм DTX-I; $n=7$ ) (Fig. $3 B$ ). Reducing outward current with either 4-AP or voltage inactivation unmasked an existing inward current that was sensitive to the $\mathrm{Na}^{+}$channel blocker TTX $[1 \mu \mathrm{M}$; data not shown (Axmacher and Miles, 2004)]. Importantly, this inward current was found to follow the outward current, in a temporal sequence that is the opposite of that reported previously in hippocampal interneurons (Fricker and Miles, 2000). This outward-inward sequence was observed in 20 of 27 neurons tested, indicating that both currents are activated in response to synaptic-like input in a sequential antagonistic manner. Thus, the outward-inward sequence of current activated during the depolarization of an EPSP is mediated by D-type $\mathrm{K}^{+}$and $\mathrm{Na}^{+}$ channels.

\section{Stochastic model predicts experimental observations}

To determine whether the presence of $I_{\mathrm{D}}$ is sufficient to reproduce our experimental observations, we implemented a multicompartment Hodgkin-Huxley model of a CA3 neuron including $I_{\mathrm{A}}$ and $I_{\mathrm{D}}$ and a stochastic $\mathrm{Na}^{+}$current (see Materials and Methods). In the presence of $I_{\mathrm{A}}$ and $I_{\mathrm{D}}$, DC current injections evoke a ramp-anddelay phenotype that is abolished when $I_{\mathrm{D}}$ is removed but not when $I_{\mathrm{A}}$ is suppressed (Fig. $4 \mathrm{~A}$ ). To test the model response to synaptic input, EPSC-like current commands were adjusted to evoke single APs at comparable latency (Fig. $4 B$ ). Concomitant with the removal of $I_{\mathrm{D}}$, there is an increase in the precision of the first AP onset time (Fig. 4C). As observed in the experiments, the membrane potential trajectory before the $\mathrm{AP}(\mathrm{dV} / \mathrm{dt})$ was slow in the presence of $I_{\mathrm{D}}$ and increased when $I_{\mathrm{D}}$ was removed (Fig. $4 D$ ). Here again, suppressing $I_{\mathrm{A}}$ had comparatively little effect on spike precision. In addition, the variation of the voltage threshold (SD $V_{\text {thresh }}$ ) for AP generation was reduced when $I_{\mathrm{D}}$ but not $I_{\mathrm{A}}$ was removed. Thus, the behavior of the model in the presence and absence of $I_{\mathrm{D}}$ reproduces our experimental findings, indicating $I_{\mathrm{D}}$ creates an outward/inward current se- 
quence that slows the voltage trajectory before an AP and subsequently reduces AP timing precision.

\section{Activity deprivation reduces the $I_{\mathrm{D}}$ current and increase spike-time precision}

Spontaneous network activity constitutes a hallmark of developing neuronal networks and organotypic hippocampal slices display in vitro increasing amounts of synaptic activity (Gähwiler et al., 1997; Johnson and Buonomano, 2007). Since $I_{\mathrm{D}}$ helps control excitability, we tested whether it might be modified during homeostatic plasticity induced by activity deprivation. Excitatory synaptic transmission was blocked with $\mathrm{Ky}(2-4 \mathrm{~mm})$ for $2 \mathrm{~d}$ to reduce the level of network activity. CA3 neurons responded to activity deprivation with a reduction in the subthreshold ramp (control, $10.1 \pm 1.2 \mathrm{mV} / \mathrm{s}$; treated, $3.9 \pm$ $0.5 \mathrm{mV} / \mathrm{ms} ; n=7 ; p=0.001$; measured 200-300 ms after the onset of a DC pulse), a leftward shift in the input-output curve (Fig. 5A) (current threshold for eliciting an AP; control: $214 \pm 8 \mathrm{pA}, n=14$; Ky treated: $103 \pm 5 \mathrm{pA}, n=13 ; p=0.001$ ), and a reduction in the latency to the first spike (supplemental Fig. S7, available at www.jneurosci.org as supplemental material) (control, $764 \pm 35 \mathrm{~ms}, n=25 \mathrm{vs}$ treated, $582 \pm 43 \mathrm{~ms}, n=13 ; p=0.0007)$. In addition, voltage-clamp recordings revealed a reduction in the amplitude of transient outward current in Ky-treated neurons compared with controls (Fig. 5B) (for steps to $0 \mathrm{mV}$ peak current in control, $733 \pm 30 \mathrm{pA}$ vs treated, $409 \pm 53 \mathrm{pA} ; n=$ $11 ; p=0.05)$. There was no obvious change in the voltage dependence or whole-cell capacitance (control, $230 \pm$ $14.1 \mathrm{pF}$; treated, $249 \pm 20.3 \mathrm{pF} ; n=10$; $p=0.46)$.

We next wanted to determine whether the activity deprivation induced decrease in current threshold and first AP latency could be, in part, caused by a reduction in D-type current. To do this, we compared the firing phenotype of control and Kytreated CA3 neurons before and after bath application of DTX-I. In control cultures, bath application of DTX-I produced a large leftward shift in the input-output curve of CA3 neurons (Fig. 5C) (DTX-I induced decrease in current threshold; $109 \pm 27 \mathrm{pA} n=7$ ), mimicking the leftward shift in response to activity deprivation, whereas in Ky-treated sister cultures, bath application of DTX-I produced almost no change $(28 \pm 9 \mathrm{pA}, n=6 \mathrm{vs} 109 \pm 27 \mathrm{pA}$, $n=7$ in control; $p=0.011)$, indicating an occlusion of the effect of DTX-I seen in control. Similar results were obtained with DTX-K (50 nM), a selective blocker of Kv1.1-containing channels (reduction in current threshold: control, $64 \pm 5 \mathrm{pA}, n=7$ vs
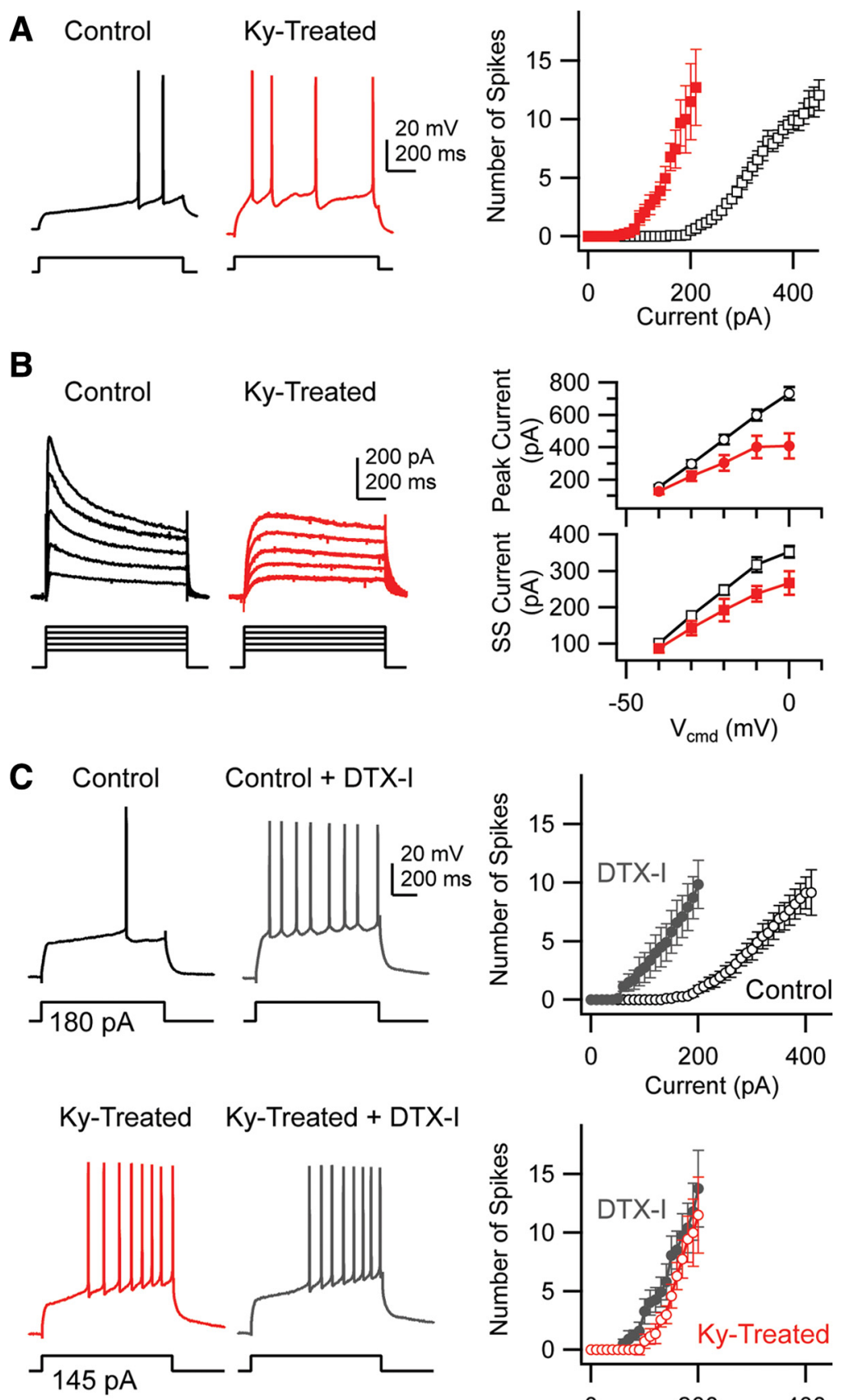

Figure 5. Activity deprivation reduces a DTX-sensitive current in CA3 neurons. $\boldsymbol{A}$, Left, Example current-clamp traces recorded in CA3 pyramidal neurons in control (black) and in Ky-treated (red) cultures. Right, Average data across groups. Ky-treated cells (red; $n=13$ ) show an increase in excitability compared with controls (black; $n=14$ ). $\boldsymbol{B}$, Left, Example voltage-clamp responses showing the reduction of the Kv currents after activity deprivation by Ky treatment. Right, Pooled $I / V$ plot showing the reduction of the peak (top plot) and sustained (bottom plot) currents measured in control versus treated cells. C, DTX-l increases the excitability of CA3 pyramidal neurons in control cultures (top row) but not in Ky-treated cultures (bottom row). Left, Representative voltage traces before (control/treated) and after application of DTX-I (control + DTX-I/treated + DTX-I). Right, Average data across groups. Note the large shift induced by DTX-I in control but not in treated cells.

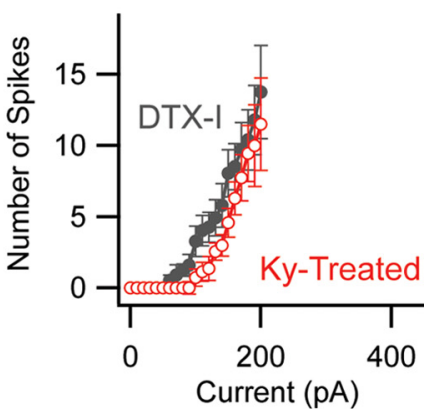

treated, $23 \pm 16 \mathrm{pA}, n=4 ; p=0.007)$. Finally, application of DTX-I to Ky-treated cultures did not further reduce the latency of the first AP (supplemental Fig. S7, available at www.jneurosci. org as supplemental material) (Ky treated, $582 \pm 43 \mathrm{~ms}, n=13 \mathrm{vs}$ $558 \pm 61 \mathrm{~ms}, n=14 ; p=0.37)$. Together, these data demonstrate that the contribution of DTX-sensitive currents in defining the current threshold and first AP latency is reduced in Ky-treated cultures, suggesting that activity deprivation reduces $I_{\mathrm{D}}$. This 
A

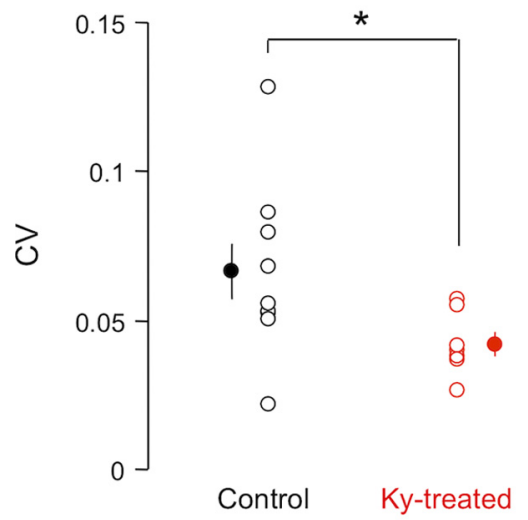

B

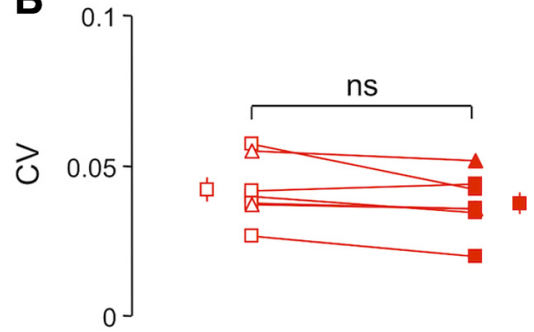

$\left.\begin{array}{l}4 \\ 3 \\ 2 \\ 1- \\ 0\end{array}\right]$

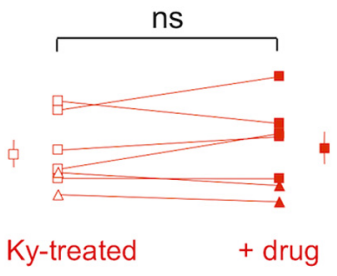

Figure 6. Spike-time precision in response to synaptic-like input is enhanced in Ky-treated neurons. $A$, Comparison of spike time precision (CV) from control and Ky-treated neurons $\left({ }^{*} p<0.05\right)$. B. Effect of D-type potassium blocker 4-AP/DTX-I on spike-time precision (top) and voltage-trajectory $\mathrm{dV} / \mathrm{dt}$ (bottom; $\mathrm{ns}, p>0.4$ ).

does not rule out activity deprivation induced changes in other ion channels (e.g., $\mathrm{Na}^{+}, \mathrm{Ca}^{2+}$, or other $\mathrm{K}^{+}$species) that could also contribute to the sculpting of excitability and spike-time precision.

\section{Activity deprivation improves spike-time precision}

Because D-type current can control spike-time precision, we wanted to determine whether spike precision was improved after activity deprivation. Single APs were evoked in CA3 neurons by an EPSC-like current (Fig. 2A). Compared with control, CA3 neurons spike-time precision was significantly improved in $\mathrm{Ky}$ treated neurons (Fig. 6A) (CV: control, $0.066 \pm 0.009, n=10$ vs Ky treated, $0.042 \pm 0.004, n=6 ; p=0.047)$. Furthermore, application of D-type current blockers $(30 \mu \mathrm{M} 4-\mathrm{AP}$ or $50 \mathrm{~nm}$ DTX-I) did not lead to an additional improvement in spike-time precision (Fig. $6 B)(\mathrm{CV}, 0.038 \pm 0.004$ vs $0.042 \pm 0.004 ; p=$ 0.428 ) or change in the membrane potential trajectory leading to the first AP (Fig. $6 B)(\mathrm{dV} / \mathrm{dt}, 1.89 \pm 0.20$ vs $1.98 \pm 0.24 ; p=$ 0.38 ). Thus, spike-time precision is improved after activity deprivation, and this enhancement occludes additional improvements in precision by DTX-I, indicating that one mechanism by which activity deprivation can enhance spike-time precision is through a reduction in $I_{\mathrm{D}}$.

\section{Development of synchronization in a hybrid CA3 network}

An intriguing question is whether $I_{\mathrm{D}}$ cannot only mediate AP precision in individual neurons but could also control network synchronization. To evaluate the effects of reducing $I_{\mathrm{D}}$ in CA3 neurons on network activity, we acutely blocked inhibitory synaptic transmission with PiTX and measured the resultant burst frequency in CA3 neurons. Bath application of PiTX produced relatively little activity in control slices (supplemental Fig. S6, available at www.jneurosci.org as supplemental material) (0.02 \pm $0.01 \mathrm{~Hz}$ ) but robust network activity in activity-deprived slices $(0.13 \pm 0.06 \mathrm{~Hz} ; n=6 ; p=0.007)$. In this set of experiments, dual recordings were performed, and in all cases, when a burst was observed in one neuron, there was always a corresponding burst in the other indicating that the bursting is a network event. In activity-deprived neurons, many synaptic and intrinsic conductances are regulated. Among them, $I_{\mathrm{D}}$ is reduced, but its pre-

cise role cannot be precisely analyzed here because all the other regulations may also improve synchronization.

To more directly examine the role of $I_{\mathrm{D}}$ in sculpting network synchronization, we constructed hybrid biological/simulated layered CA3 networks (Reyes, 2003) and compared the development of network synchrony as a function of layer between control and activity-deprived slices. Thus, the role of postsynaptic ion channels on network synchrony can be tested independently of changes in synaptic efficacy. The hybrid network was seeded in layer 0 with 200 simulated neurons all firing APs at an average rate of $10 \mathrm{~Hz}$ with no correlation between neurons (Fig. 7A). The binary AP output of each layer 0 neuron was convolved with an EPSC template that then became the presynaptic input for the next layer. For subsequent layers, one whole-cell recording was used to construct the remaining network. Each CA3 neuron in the network received as input the sum of the synaptic input from $40 \%$ of a randomly selected population of presynaptic neurons. The binary AP output of each real neuron was calculated from the actual response, and this was again convolved and became input to the next layer.

In activity-deprived slices, synchronous network activity developed in earlier layers compared with control (Fig. 7B). Comparing the spiking activity of the population of neurons within a layer reveals that, even in the fourth layer where the activity seems highly synchronous, treated slices are more synchronous than control (Fig. 7C). To rule out the possibility that an overall increase in excitability could explain the increase in synchrony of treated networks, we matched the evoked firing rate between conditions (control, $9.3 \pm 0.6 \mathrm{~Hz}$; treated, $9.9 \pm 0.4 \mathrm{~Hz} ; p=0.5$ ) by monitoring the number of spikes evoked by each trial during the experiment. To quantify the level of synchrony within a layer, we calculated the normalized $\mathrm{CCH}$ for all pairwise binary spike trains (see Materials and Methods). The normalized CCH quantifies the strength of correlation of APs across a population of neurons; for any given time bin (bin width, $0.2 \mathrm{~ms}$ ), the $\mathrm{CCH}$ takes a value of 0 if no two neurons are firing and a value of 1 if all neurons are firing. The normalized CCH confirms that activitydeprived slices develop synchrony in earlier layers compared with control, as the central peak of the $\mathrm{CCH}$ became more powerful (Fig. $7 D$ ). We also calculated the normalized $\mathrm{CCH}$ for a family of bin widths (0.2-500 ms in $0.2 \mathrm{~ms}$ steps) and verified there was no departure from a linear increase in the $\mathrm{CCH}$ as a function of bin width (Wang and Buzsaki, 1996), indicating the activity in a layer is not oscillating through time but instead is synchronous across the population of neurons.

To strengthen our conclusion, we next determined whether reducing $I_{\mathrm{D}}$ in control slices with voltage inactivation $(-50 /-55$ $\mathrm{mV}$ ) would mimic the improved synchronization found in activity-deprived slices. Again, we constructed a control hybrid network and tested the role of voltage inactivation of $\mathrm{Kv}$ currents on network synchrony (Fig. 8A). Comparing the synchronization as a function of layer in these two conditions, we found inactivation of $\mathrm{Kv}$ currents in control slices creates a network that synchronizes in earlier layers. Consistent with the contribution of $I_{\mathrm{D}}$ to network synchrony, the improvement in synchronization 
A

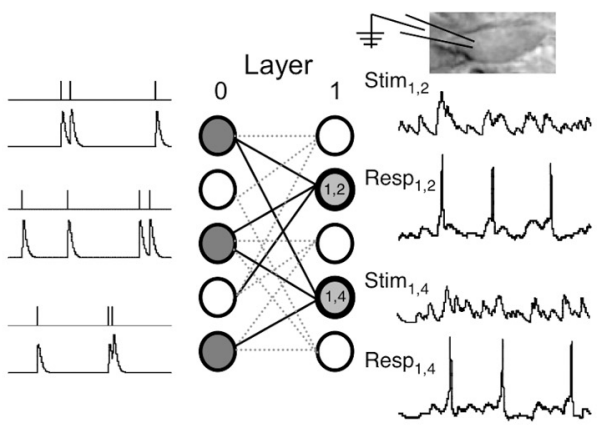

B

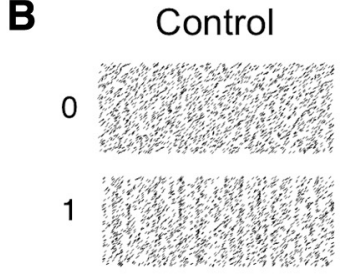

㐫 2
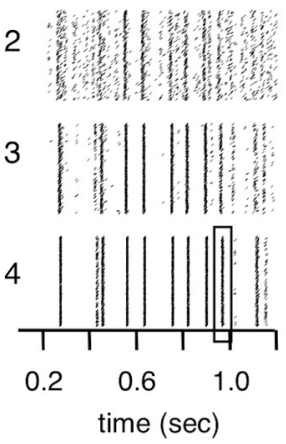

C

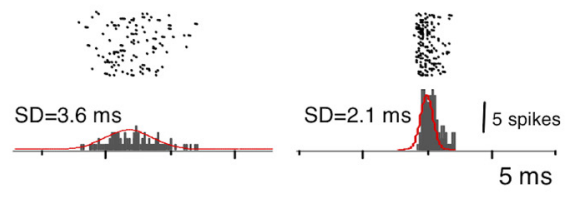

D
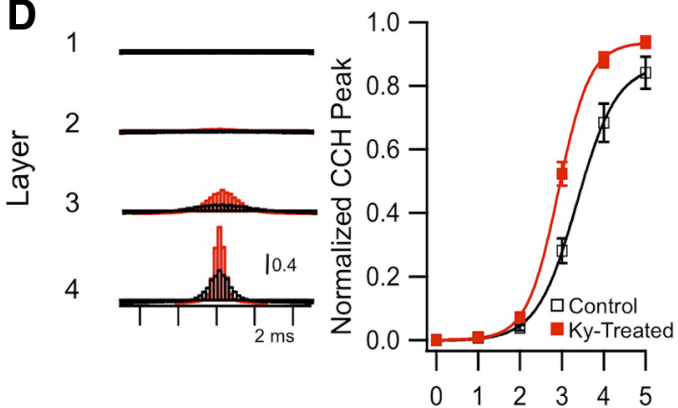

Figure 7. Activity-deprived slices synchronize in earlier layers of a hybrid network model.A, Schematic showing the construction of a hybrid network. The network is seeded with a modeled layer firing random APs at $10 \mathrm{~Hz}$ (left column, layer 0 ). Layer 0 makes random connections with the next layer (right column, layer 1). Each neuron in layer 1 is stimulated with a current, which is the summation of a random selection of the input from layer $0 . B$, Example spike raster plots showing the development of synchrony across neurons in successive layers. Treated slices develop more synchronization beginning with layer 2. $\boldsymbol{C}$, Expanded plot of spike times in the boxed region of the fourth layer $(\boldsymbol{B})$ showing more synchrony across neurons in treated versus control. $\boldsymbol{D}$, The central peak in the normalized CCH histogram (left) increased after activity deprivation (red). The plot of the peak CCH versus layer with Sigmoid fits (right; $n=7$; for fourth, $p<0.05$ ) is shown.

was maximal at the beginning of the EPSC train after voltage inactivation (Fig. $8 \mathrm{~B}$ ). In fact, in control conditions the amplitude of the sliding normalized $\mathrm{CCH}$ increased progressively from the onset of the EPSC train to its end, consistent with the slow
A Control
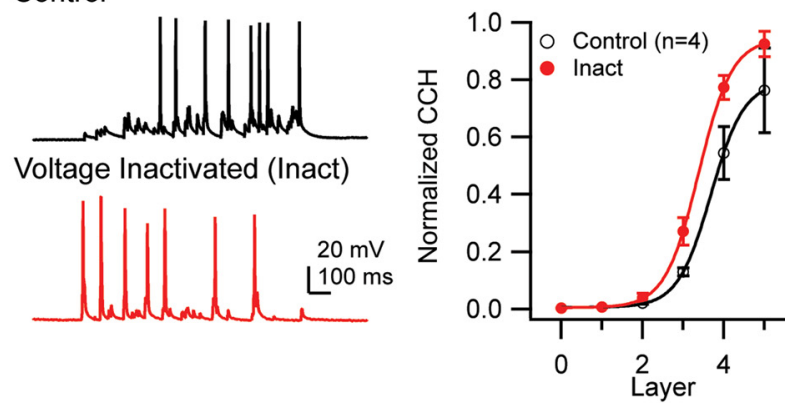

B Control

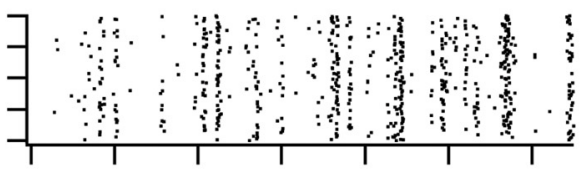

Voltage Inactivated (Inact)

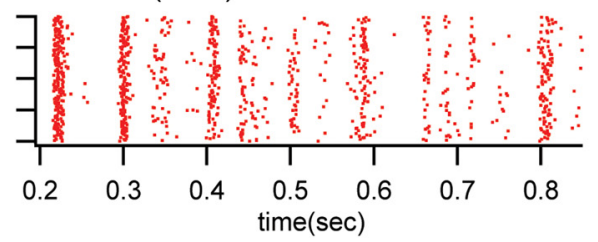

C
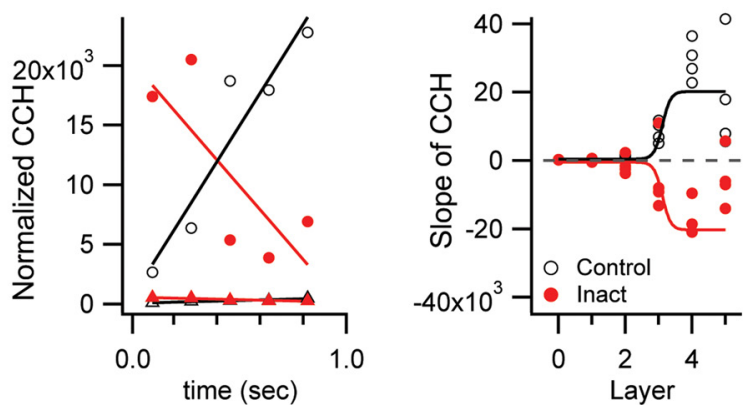

Figure 8. Voltage inactivation of Kv currents increases the development of network synchrony. A, Left, Example traces of the response of fourth layer neurons in control $(-70 \mathrm{mV})$ and voltage inactivation $(-51 \mathrm{mV})$. Right, Normalized $\mathrm{CCH}$ as a function of layer for matched control versus voltage-inactivation networks. $\boldsymbol{B}$, Spike raster plots of neurons in the fourth layer showing an increase in synchrony over time for control and a decrease in synchrony over time for voltage inactivation (Inact). C, Left, Sliding CCH (200 ms window) showing the evolution of synchrony through time. The control layer (black) develops synchrony with time, and the voltage-inactivated layer (red) starts with maximal synchrony and decreases. Right, Slope of sliding $\mathrm{CCH}$ as a function of layer, showing the evolution of an increase in slope for control (black) and a decrease in voltage-inactivated layer (red).

inactivation of $I_{\mathrm{D}}$ during the train. In contrast, no increase in synchrony was observed when $I_{\mathrm{D}}$ was already inactivated (Fig. $8 C$ ). Together, these data support the fact that $I_{\mathrm{D}}$ is a key determinant of network synchronization in the CA3 region, limiting synchrony during the onset of an EPSC train.

\section{Discussion}

Our study demonstrates that the sequence of outward D-type $\mathrm{K}^{+}$ followed by inward $\mathrm{Na}^{+}$current increases temporal jitter in the generation of APs in individual neurons that can, in turn, create a network less susceptible to the production of synchronized activity. Furthermore, we show that activity-dependent homeostatic plasticity may adjust $\mathrm{D}$-type $\mathrm{K}^{+}$currents in CA3 pyramidal neurons to improve spike-time precision and sculpt network synchrony to within physiological bounds. Thus, homeostatic plasticity of neuronal excitability is not limited to the control of 
the input-output function but can also determine spike timing and network activity.

Using both whole-cell recording and stochastic modeling, we show that $I_{\mathrm{D}}$ introduces a significant jitter in AP generation. $I_{\mathrm{D}}$ is activated during long depolarizing voltage steps and EPSP waveforms, in agreement with previous findings (Mitterdorfer and Bean, 2002). The presence of $I_{\mathrm{D}}$ desynchronizes APs in response to both DC and EPSC waveforms, whereas the pharmacological removal of $I_{\mathrm{D}}$ improves AP precision. To define the precise temporal activation of currents that mediate this modulation of AP timing, we demonstrate that there is a sequence of $\mathrm{K}^{+}$-mediated outward followed by $\mathrm{Na}^{+}$-mediated inward currents evoked in response to EPSP-like voltage commands. This sequence of outward-inward current slows the voltage trajectory before the spike and effectively introduces jitter in the generation of APs.

Control of network synchrony by ion channels may be of particular importance in the pyramidal CA3 network, as its high level of interconnection can produce synchronous activity associated with epilepsies (Traub and Wong, 1982). The control of network synchrony by $I_{\mathrm{D}}$ could also be of general importance in other brain regions where the ramp-and-delay phenotype is expressed in principal neurons (Adam et al., 1999; Martina et al., 1999; Russier et al., 2003) or interneurons (Goldberg et al., 2008).

\section{Ion channels control the temporal precision of APs: a novel law}

It is well established that postsynaptic ion channels distributed in the dendrite, soma, and axon both spatially and temporally sculpt synaptic input into AP output (Debanne, 2004; Frick and Johnston, 2005; Zhang and Linden, 2003). Yet, the precise rules by which this is achieved may differ across brain regions and cell types. For example, postsynaptic inward and outward currents determine AP precision in hippocampal neurons (Fricker and Miles, 2000; Axmacher and Miles, 2004). Compared with hippocampal CA1 pyramidal neurons that primarily exhibit inward currents and low precision, CA1 interneurons display high AP precision, mediated by a sequence of inward followed by outward current (Fricker and Miles, 2000). The sequence of current is particularly critical here since the outward current developing after the inward current shortens the EPSP duration and reduces the time window in which an AP can be elicited. This rule of Fricker and Miles (2000) has been verified in many cases including improved EPSP-spike precision when the EPSP duration is reduced through inhibition by $\mathrm{GABA}_{\mathrm{A}}$ receptors (Pouille and Scanziani, 2001), recruitment of h-channels (Campanac et al., 2008), or suppression of persistent sodium current (Vervaeke et al., 2006). For the same reasons, precision is higher in mature neurons because input resistance and membrane time constant are decreased and therefore EPSPs display fast decay kinetics (Cathala et al., 2003).

Here we describe a different mechanism, where the outward current occurs before the inward current and competes with the generation of an AP and thus introduces jitter in AP generation (Fig. 9). Thus, this sequence is in the opposite order of that which can improve the precision of EPSP-spike coupling (Fricker and Miles, 2000; Pouille and Scanziani, 2001; Axmacher and Miles, 2004). The key parameter here is the rate of depolarization leading to the generation of an AP. We show in both experiments, and a stochastic model, that the presence of $I_{\mathrm{D}}$ decreases the rate of depolarization in the last $\sim 10 \mathrm{~ms}$ before spike generation and degrades the fidelity of AP generation. Removing $I_{\mathrm{D}}$ increases this voltage trajectory and, in turn, improves the fidelity of AP generation. This novel rule may also account for the SK-dependent

\section{Fricker and Miles}

Cudmore et al.
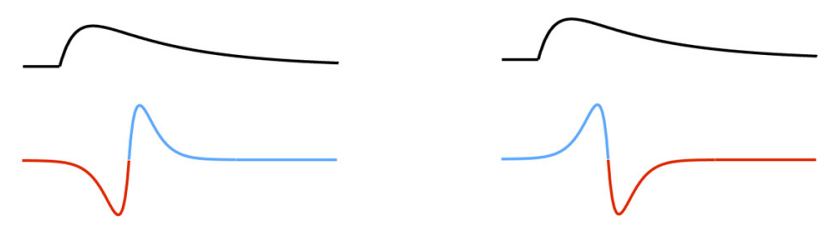

Inward + Outward

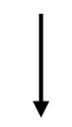

High precision (faster EPSP decay)
Outward + Inward

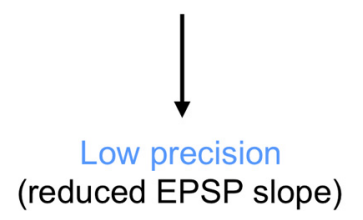

Figure 9. Two rules by which outward $\mathrm{K}^{+}$current can control AP precision. As described by Fricker and Miles (2000), in the left column the sequence of inward followed by outward current produces low AP jitter because the outward current shortens the EPSP decay and prevents late AP generation. Reducing outward $\mathrm{K}^{+}$current in this case will introduce more jitter. In thr right column, the sequence of outward followed by inward current introduces AP jitter by delaying the first spike and reducing the membrane potential trajectory before the spike. Reducing outward $\mathrm{K}^{+}$current in this case improves AP precision.

precision of the second spike (Sourdet et al., 2003) (but see Wolfart et al., 2001; Deister et al., 2009) and for the $I_{\mathrm{h}}$-dependent control of rebound spike (Campanac et al., 2008), since these currents also control the voltage trajectories leading to spike generation. Furthermore, this rule is compatible with the enhanced precision produced by the amplification of fast afterhyperpolarizations by autaptic contacts in neocortical interneurons (Bacci and Huguenard, 2006). The mechanism that governs slope-dependent precision is not fully, elucidated but it could be related to the fact that spike threshold is not a fixed point but rather a voltage range because of stochastic properties of ion channels (Schneidman et al., 1998; White et al., 2000). Crossing the spike threshold with a slow rate would trigger a spike with very high temporal dispersion. In contrast, fast depolarization would produce a spike with low temporal dispersion.

\section{$I_{\mathrm{D}}$ reduces network synchrony}

We show here that the strength of $I_{\mathrm{D}}$ can control the development of network synchrony. We first performed pharmacological experiments to compare the frequency of synchronous bursting in response to blocking synaptic inhibition. In control slices, blocking GABAergic inhibition produced synchronized bursts at low frequency, whereas the same experiments in Ky-treated cultures increased bursting frequency by a factor of 6 . These data suggest that $I_{\mathrm{D}}$ acts as an "intrinsic brake" on cellular excitability and network synchronization. However, in these experiments an increase in bursting could also be mediated by an overall increase in excitability or by the differential scaling of excitatory and inhibitory synaptic transmission (Turrigiano and Nelson, 2004).

With the use of hybrid networks of biological CA3 neurons constructed with feed-forward architecture, we show that the presence of $I_{\mathrm{D}}$ decreased both the amount and the rate of development of network synchrony. In contrast, hybrid networks of Ky-treated or voltage-inactivated CA3 neurons exhibit both faster and more powerful synchronization. Together, the results from the hybrid network experiments provide strong evidence that the expression level of $I_{\mathrm{D}}$ is sufficient to mediate network 
synchronization. The hybrid network approach is especially appealing for three reasons. First, the hybrid model allows synapses to be modeled while the neurons are real. Thus, the role of voltage-gated membrane currents on the properties of the network can be dissected without contamination introduced by changes in synaptic properties (Turrigiano and Nelson, 2004). Second, the pyramidal CA3 network is highly interconnected and considered to be a fairly homogeneous population, thus the feedforward architecture and the use of a few neurons to construct a hybrid network is appropriate. Finally, the topology of the hybrid network, a multilayer feed-forward network, is known to eventually lead to synchronous population activity (Reyes, 2003) allowing for the comparison of synchrony between different experimental conditions.

\section{Homeostatic plasticity and network synchrony}

During the first weeks in vitro, the hippocampal slice culture is undergoing axonal sprouting and synaptogenesis, resulting in a continual increase in synaptic activity that parallels in vivo development (Gähwiler et al., 1997). Thus, in control slices, the presence of $I_{\mathrm{D}}$, which reduces spike precision and network synchrony, is already a homeostatic response that acts as a safety net against excess network synchronization. By reducing spontaneous network activity with chronic activity blockade (Ky treatment), we have pushed the homeostatic mechanisms in the opposite direction, reducing $I_{\mathrm{D}}$ to promote network synchrony. In fact, compared with control cultures, DTX-I or DTX-K had virtually no effect on the excitability of CA3 pyramidal neurons, indicating that downregulation of D-type current, presumably mediated by Kv1.1 channels, represents a main target of activity deprivation. Our results are consistent with other recent findings showing that activity deprivation produces a decrease in the expression of Kv1.1 channels in hippocampal (Grosse et al., 2000; Raab-Graham et al., 2006) and auditory brainstem neurons (Lu et al., 2004).

Studies examining homeostatic plasticity have primarily focused on measurements in individual neurons, yet given the fact that homeostasis can be a global event affecting all members in a network begs the question as to the network effect of homeostasis. Homeostatic plasticity has previously been implicated in the restoration of rhythmic network activity in invertebrates (Turrigiano et al., 1994) and the spinal cord (Galante et al., 2001). Studies using in vivo sensory deprivation (Maffei and Turrigiano, 2008), organotypic slice culture (Buckby et al., 2006; Karmarkar and Buonomano, 2006; Kim and Tsien, 2008), and computational modeling (Frohlich et al., 2008) have begun to explore the network effects of homeostasis. Here we show that the presence of an ionic conductance cannot only control the precision of the generation of an AP at the scale of single principal neurons but can also determine network synchrony, endowing voltagedependent ion channels with increasing functional significance. Given that the sequence of outward followed by inward currents can sculpt precision in individual neurons, it is not surprising that a population of neurons possessing this sequence could modulate spiking activity at the network level. We assume this current sequence operates in conjunction with the sequence of synaptic excitation and inhibition (Pouille and Scanziani, 2001) to define the final network state. It will be interesting to determine whether these two processes work in parallel, in sequence, or synergistically. Given the enormous variety of ion channel species, the pathways for their plasticity, and their precise localization (Vacher et al., 2008), ion channels may serve as a substrate by which network activity can be modified and maintained.

\section{References}

Adam TJ, Schwarz DW, Finlayson PG (1999) Firing properties of chopper and delay neurons in the lateral superior olive of the rat. Exp Brain Res 124:489-502.

Axmacher N, Miles R (2004) Intrinsic cellular currents and the temporal precision of EPSP-action potential coupling in CA1 pyramidal cells. J Physiol 555:713-725.

Bacci A, Huguenard JR (2006) Enhancement of spike-timing precision by autaptic transmission in neocortical inhibitory interneurons. Neuron 49:119-130.

Bekkers JM, Delaney AJ (2001) Modulation of excitability by alphadendrotoxin-sensitive potassium channels in neocortical pyramidal neurons. J Neurosci 21:6553-6560.

Boudkkazi S, Carlier E, Ankri N, Caillard O, Giraud P, Fronzaroli-Molinieres L, Debanne D (2007) Release-dependent variations in synaptic latency: a putative code for short- and long-term synaptic dynamics. Neuron 56:1048-1060.

Buckby LE, Jensen TP, Smith PJ, Empson RM (2006) Network stability through homeostatic scaling of excitatory and inhibitory synapses following inactivity in CA3 of rat organotypic hippocampal slice cultures. Mol Cell Neurosci 31:805-816.

Buonomano DV (2005) A learning rule for the emergence of stable dynamics and timing in recurrent networks. J Neurophysiol 94:2275-2283.

Campanac E, Gastrein P, Cudmore RH, Ankri N, Debanne D (2008) Hyperpolarization-activated cationic current (Ih) organizes temporal activity in cortical circuits in vitro. FENS Abstr 4:47.46.

Cathala L, Brickley S, Cull-Candy S, Farrant M (2003) Maturation of EPSCs and intrinsic membrane properties enhances precision at a cerebellar synapse. J Neurosci 23:6074-6085.

Cudmore RH, Turrigiano GG (2004) Long-term potentiation of intrinsic excitability in LV visual cortical neurons. J Neurophysiol 92:341-348.

Debanne D (2004) Information processing in the axon. Nat Rev Neurosci 5:304-316.

Debanne D, Guérineau NC, Gähwiler BH, Thompson SM (1995) Physiology and pharmacology of unitary synaptic connections between pairs of cells in areas CA3 and CA1 of rat hippocampal slice cultures. J Neurophysiol 73:1282-1294.

Debanne D, Boudkkazi S, Campanac E, Cudmore RH, Giraud P, FronzaroliMolinieres L, Carlier E, Caillard O (2008) Paired-recordings from synaptically coupled cortical and hippocampal neurons in acute and cultured brain slices. Nat Protoc 3:1559-1568.

Deister CA, Chan CS, Surmeier DJ, Wilson CJ (2009) Calcium-activated SK channels influence voltage-gated ion channels to determine the precision of firing in globus pallidus neurons. J Neurosci 29:8452-8461.

Desai NS, Rutherford LC, Turrigiano GG (1999) Plasticity in the intrinsic excitability of cortical pyramidal neurons. Nat Neurosci 2:515-520.

Fox RF (1997) Stochastic versions of the Hodgkin-Huxley equations. Biophys J 72:2068-2074

Frick A, Johnston D (2005) Plasticity of dendritic excitability. J Neurobiol 64:100-115.

Fricker D, Miles R (2000) EPSP amplification and the precision of spike timing in hippocampal neurons. Neuron 28:559-569.

Frohlich F, Bazhenov M, Sejnowski TJ (2008) Pathological effect of homeostatic synaptic scaling on network dynamics in diseases of the cortex. J Neurosci 28:1709-1720.

Gähwiler BH, Capogna M, Debanne D, McKinney RA, Thompson SM (1997) Organotypic slice cultures: a technique has come of age. Trends Neurosci 20:471-477.

Galante M, Avossa D, Rosato-Siri M, Ballerini L (2001) Homeostatic plasticity induced by chronic block of AMPA/kainate receptors modulates the generation of rhythmic bursting in rat spinal cord organotypic cultures. Eur J Neurosci 14:903-917.

Goldberg EM, Clark BD, Zagha E, Nahmani M, Erisir A, Rudy B (2008) K+ channels at the axon initial segment dampen near-threshold excitability of neocortical fast-spiking GABAergic interneurons. Neuron 58:387-400.

Grosse G, Draguhn A, Hohne L, Tapp R, Veh RW, Ahnert-Hilger G (2000) Expression of Kvl potassium channels in mouse hippocampal primary cultures: development and activity-dependent regulation. J Neurosci 20:1869-1882.

Guan D, Lee JC, Higgs MH, Spain WJ, Foehring RC (2007) Functional roles of Kv1 channels in neocortical pyramidal neurons. J Neurophysiol 97:1931-1940. 
Hemond P, Epstein D, Boley A, Migliore M, Ascoli GA, Jaffe DB (2008) Distinct classes of pyramidal cells exhibit mutually exclusive firing patterns in hippocampal area CA3b. Hippocampus 18:411-424.

Hines ML, Carnevale NT (2000) Expanding NEURON's repertoire of mechanisms with NMODL. Neural Comput 12:995-1007.

Johnson HA, Buonomano DV (2007) Development and plasticity of spontaneous activity and Up states in cortical organotypic slices. J Neurosci 27:5915-5925.

Karmarkar UR, Buonomano DV (2006) Different forms of homeostatic plasticity are engaged with distinct temporal profiles. Eur J Neurosci 23:1575-1584.

Kim J, Tsien RW (2008) Synapse-specific adaptations to inactivity in hippocampal circuits achieve homeostatic gain control while dampening network reverberation. Neuron 58: 925-37.

Kole MH, Letzkus JJ, Stuart GJ (2007) Axon initial segment Kv1 channels control axonal action potential waveform and synaptic efficacy. Neuron 55:633-647.

Locke RE, Nerbonne JM (1997) Three kinetically distinct $\mathrm{Ca}_{2}{ }^{+}$. independent depolarization-activated $\mathrm{K}^{+}$currents in callosal-projecting rat visual cortical neurons. J Neurophysiol 78:2309-2320.

Lu Y, Monsivais P, Tempel BL, Rubel EW (2004) Activity-dependent regulation of the potassium channel subunits Kv1.1 and Kv3.1. J Comp Neurol 470:93-106.

Luthi A, Gähwiler BH, Gerber U (1996) A slowly inactivating potassium current in CA3 pyramidal cells of rat hippocampus in vitro. J Neurosci 16:586-594.

Maffei A, Turrigiano GG (2008) Multiple modes of network homeostasis in visual cortical layer 2/3. J Neurosci 28:4377-4384.

Martina M, Royer S, Pare D (1999) Physiological properties of central medial and central lateral amygdala neurons. J Neurophysiol 82:1843-1854.

Miller MN, Okaty BW, Nelson SB (2008) Region-specific spike-frequency acceleration in layer 5 pyramidal neurons mediated by Kv1 subunits. J Neurosci 28:13716-13726.

Mitterdorfer J, Bean BP (2002) Potassium currents during the action potential of hippocampal CA3 neurons. J Neurosci 22:10106-10115.

Pouille F, Scanziani M (2001) Enforcement of temporal fidelity in pyramidal cells by somatic feed-forward inhibition. Science 293:1159-1163.

Raab-Graham KF, Haddick PC, Jan YN, Jan LY (2006) Activity- and mTOR-dependent suppression of Kv1.1 channel mRNA translation in dendrites. Science 314:144-148.

Reyes AD (2003) Synchrony-dependent propagation of firing rate in iteratively constructed networks in vitro. Nat Neurosci 6:593-599.

Russier M, Carlier E, Ankri N, Fronzaroli L, Debanne D (2003) A-, T-, and
H-type currents shape intrinsic firing of developing rat abducens motoneurons. J Physiol 549:21-36.

Schneidman E, Freedman B, Segev I (1998) Ion channel stochasticity may be critical in determining the reliability and precision of spike timing. Neural Comput 10:1679-1703.

Schoppa NE, Westbrook GL (1999) Regulation of synaptic timing in the olfactory bulb by an A-type potassium current. Nat Neurosci 2:11061113.

Shu Y, Yu Y, Yang J, McCormick DA (2007) Selective control of cortical axonal spikes by a slowly inactivating $\mathrm{K}+$ current. Proc Natl Acad Sci U S A 104:11453-11458.

Sourdet V, Russier M, Daoudal G, Ankri N, Debanne D (2003) Long-term enhancement of neuronal excitability and temporal fidelity mediated by metabotropic glutamate receptor subtype 5. J Neurosci 23:10238-10248.

Stoppini L, Buchs PA, Muller D (1991) A simple method for organotypic cultures of nervous tissue. J Neurosci Methods 37:173-182.

Storm JF (1988) Temporal integration by a slowly inactivating K+ current in hippocampal neurons. Nature 336:379-381.

Traub RD, Wong RK (1982) Cellular mechanism of neuronal synchronization in epilepsy. Science 216:745-747.

Turrigiano G, Abbott LF, Marder E (1994) Activity-dependent changes in the intrinsic properties of cultured neurons. Science 264:974-977.

Turrigiano GG, Nelson SB (2004) Homeostatic plasticity in the developing nervous system. Nat Rev Neurosci 5:97-107.

Vacher H, Mohapatra DP, Trimmer JS (2008) Localization and targeting of voltage-dependent ion channels in mammalian central neurons. Physiol Rev 88:1407-1447.

Vervaeke K, Hu H, Graham LJ, Storm JF (2006) Contrasting effects of the persistent $\mathrm{Na}+$ current on neuronal excitability and spike timing. Neuron 49:257-270.

Wang XJ, Buzsaki G (1996) Gamma oscillation by synaptic inhibition in a hippocampal interneuronal network model. J Neurosci 16:6402-6413.

White JA, Rubinstein JT, Kay AR (2000) Channel noise in neurons. Trends Neurosci 23:131-137.

Wolfart J, Neuhoff H, Franz O, Roeper J (2001) Differential expression of the small-conductance, calcium-activated potassium channel SK3 is critical for pacemaker control in dopaminergic midbrain neurons. J Neurosci 21:3443-3456.

Wu RL, Barish ME (1992) Two pharmacologically and kinetically distinct transient potassium currents in cultured embryonic mouse hippocampal neurons. J Neurosci 12:2235-2246.

Zhang W, Linden DJ (2003) The other side of the engram: experiencedriven changes in neuronal intrinsic excitability. Nat Rev Neurosci 4:885900 . 